\title{
Adrenaline dacryolith: detection by ultrasound examination of the nasolacrimal duct
}

\author{
JOHN A BRADBURY,' IAN G RENNIE,' AND M ANDREW PARSONS ${ }^{2}$
}

From the Departments of ${ }^{1}$ Ophthalmology and ${ }^{2}$ Pathology, University of Sheffield

SUMMARY A 73-year-old woman on topical pilocarpine and adrenaline for chronic simple glaucoma for three years presented with a mass in the medial canthus of the right eye. Although dacryocystography showed a dilated and partially obstructed nasolacrimal system, ultrasound examination was able to demonstrate a mass in the nasolacrimal duct. At operation a black dacryolith was found, of the diameter predicted by ultrasound. Histological examination of the dacryolith suggested its derivation from breakdown products of adrenaline.

Ultrasound examination of the nasolacrimal drainage system has been shown to be of value when the system is dilated in cases such as a mucocoele or acute dacryocystitis. It is of limited value in functional disorders, where the passage of fluid through the nasolacrimal system is slowed, producing a minimally dilated system. ${ }^{1}$ No reports so far have illustrated the ability of ultrasound to demonstrate a mass in the nasolacrimal duct. This case illustrates the use of ultrasound in the detection of a solid mass in the nasolacrimal drainage system.

\section{Case history}

A 73-year-old retired nurse presented with chronic open-angle glaucoma in 1981, and she was treated with topical adrenaline $1 \%$ twice daily to both eyes. Four months later her intraocular pressures were found to be uncontrolled, and pilocarpine $4 \%$ four times daily was added to her medication to both eyes. In 1981 a left trabeculectomy was performed because of poor medical control. Pilocarpine and adrenaline were continued in the right eye. Because of travelling difficulties, from December 1981 her follow-up was continued at another unit. In 1986 she returned to this unit having suffered four episodes, over the previous eight months, of severe right-sided epiphora associated with a swelling over the right nasolacrimal duct. She also noted the presence of a rusty discharge down the right nostril and reflux of this fluid into

Correspondence to Mr J A Bradbury, Department of Ophthalmology, Leeds General Infirmary, Great George Street, Leeds LS1 3EX. her right conjunctival sac. In between these acute episodes the epiphora and a slight swelling at the medial canthus persisted but were less troublesome. Her right nasolacrimal duct had been irrigated during one of these acute episodes, which had caused some resolution of her symptoms.

On examination a firm mass was palpable over the right lacrimal fossa extending slightly above the medial palpebral ligament. When the nasolacrimal system was cannulated through the lower punctum, the sac was entered easily. Irrigation with saline produced dilatation of the sac and a reflux of rust stained fluid through the upper punctum into the conjunctival sac. Cytological and microbiological examination of this fluid failed to show any abnormal cells or micro-organisms. The patient was unable to detect the passage of any fluid into the nasopharynx during irrigation.

A right dacryocystogram was performed (Fig. 1), which showed filling of superior and inferior canaliculi; contrast fluid drained into the lacrimal sac, where it collected in irregular, globular pools. No contrast fluid was seen to enter the nasopharynx. This suggested a chronic obstruction of the nasolacrimal duct causing dilatation of the nasolacrimal sac.

\section{ULTRASONOGRAPHY}

Ultrasonography of the right nasolacrimal duct was performed with a Sonumetric Ocuscan 400 hand-held ultrasound scanner with a $40^{\circ}$ sector $10 \mathrm{MHz}$ probe, set at $1550 \mathrm{~m} / \mathrm{s}$ velocity and with an attenuation of $21 \mathrm{~dB}$.

With the patient in a prone position the ultrasound probe was held against the lacrimal fossa at an angle 
Fig. 1 Right dacryocystogram. The contrast filled the superior and inferior canaliculi and drained into the lacrimal sac collecting in irregular globular pools. No contrast was seen to enter the nasopharynx.

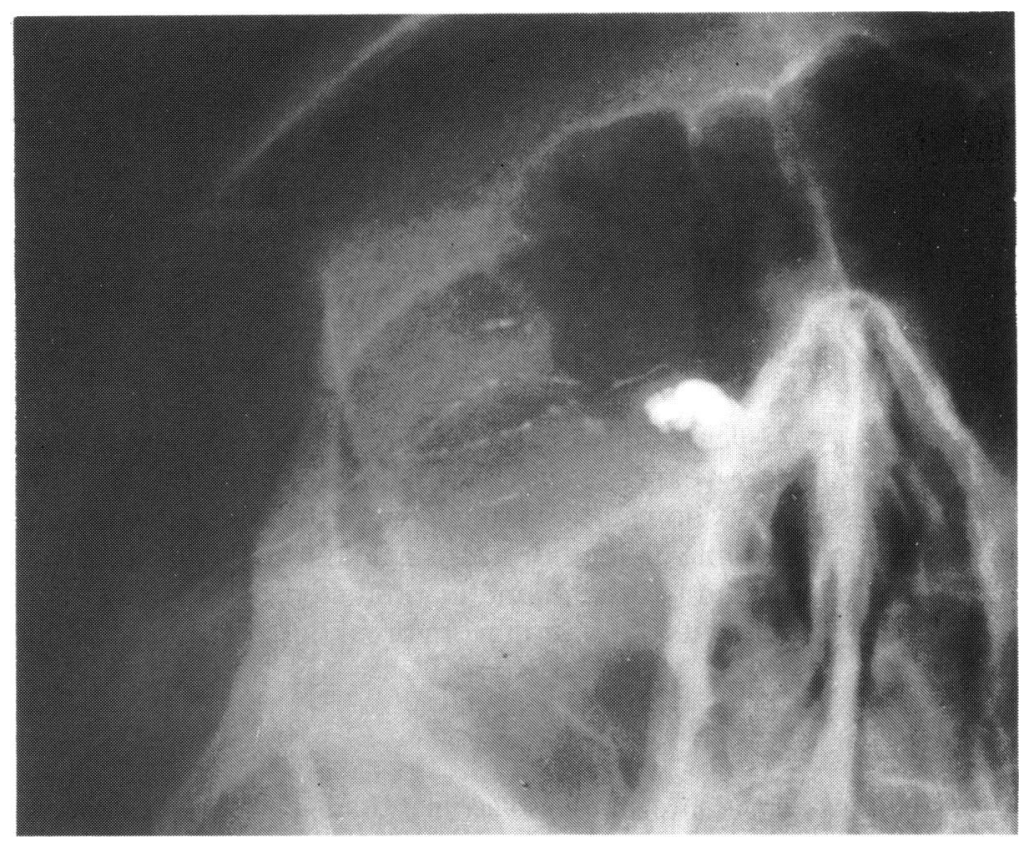

of $45^{\circ}$ to the vertical to obtain both A and B scan images, which were photographed with a Polaroid camera.

The ultrasound examination showed a round mass in a dilated nasolacrimal sac (Fig. 2). The lesion had a poorly defined acoustic edge, with high internal reflectivity, and was not obviously attached to the wall of the sac, which had a normal appearance. The

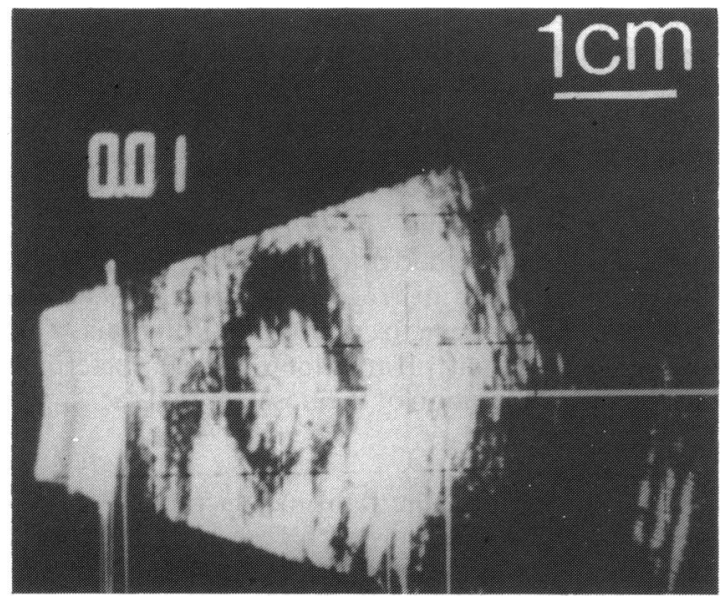

Fig. 2 Ultrasound of right nasolacrimal sac: There is a mass in a dilated nasolacrimal sac. The mass has a poorly defined acoustic edge and high internal reflectivity. It is not attached to the wall of the sac. patient was admitted for exploration of the lacrimal sac under a general anaesthetic.

A slightly larger than standard dacryocystorhinostomy approach was employed to expose an enlarged, but otherwise normal looking lacrimal sac. A vertical incision was made into the sac; it revealed a solid black mass in its posterior-inferior portion, which extended down into the nasolacrimal duct. This lesion was removed in one piece with forceps (Fig. 3) to expose a grossly enlarged nasolacrimal duct. The posterior wall of the lacrimal sac looked slightly reddened and oedematous. A small biopsy was taken of this area and bacteriological swabs were taken for culture. A standard dacryocystorhinostomy was then performed.

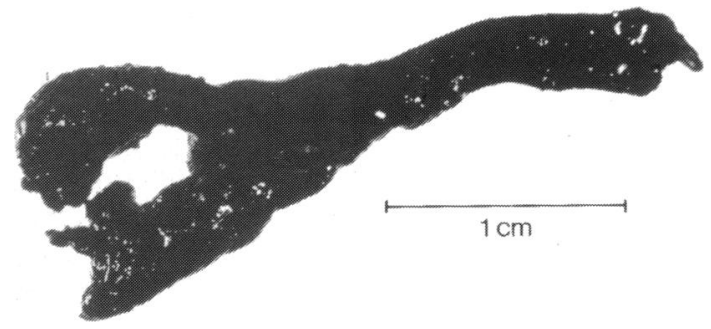

Fig. 3 Adrenaline dacryolith. The dacryolith extended inferiorly into the nasolacrimal duct and superiorly into both superior and inferior canaliculi. 


\section{HISTOLOGY}

The dacryolith was black, $4.5 \mathrm{~cm}$ long and $0.75 \mathrm{~cm}$ in maximum diameter. Histologically it consisted of amorphous eosinophilic material, which was both argentaffin and argyrophilic positive and was thus consistent with a dacryolith containing a high proportion of degraded adrenaline. Histology of the sac biopsy revealed a chronically inflamed mucous epithelium with no evidence of infection or malignancy. Bacteriological culture of the swab taken from the lacrimal sac revealed a scanty growth of Staphylococcus epidermidis.

\section{Discussion}

Diagnostic ultrasound of the nasolacrimal system has been found to be useful in assessing the size of the sac and duct, the condition of their walls, and the type of fluid in the sac, for example, pus or mucus. It may prove to be of particular use in the diagnosis of solid lesions such as dacryoliths, tumours, and granulomas, which, though less common, can be difficult to diagnose preoperatively. Ultrasound may prove a useful adjunct to dacryocystorhinostomy in such cases.

Dacryoliths are not uncommon if looked for carefully. Indeed Wilkins and Pressly ${ }^{2}$ in their series of 94 dacryocystorhinostomies reported an overall incidence of $18 \%$. In 25 cases of dacryoliths collected by Jones ${ }^{3}$ epiphora was the commonest symptom followed by mucopurulent discharge. As in this case, irrigation of the lacrimal system may afford temporary relief, perhaps due to fragmentation of the dacryolith.

Both Jones ${ }^{3}$ and Wilkins and Pressly ${ }^{2}$ found the majority of patients to be below 50 years of age. In neither series were dacryoliths found to be caused by topical adrenaline therapy, which must remain an uncommon cause of nasolacrimal duct obstruction. Spaeth $^{4}$ describes three cases of dacryoliths, in patients with chronic simple glaucoma, who were treated with $2 \%$ adrenaline drops for at least one year. In all cases irrigation through the lower puncta caused the presentation of a dark plug into the nose or nasopharynx, which relieved the patient's symptoms. Analysis of the dark plug showed this to be derived from adrenaline.

\section{References}

1 Montanara A, Mannino G, Contestabile M. Macrodacryocystography and echography in diagnosis of disorders of the lacrimal pathways. Surv Ophthalmol 1983; 28: 33-41.

2 Wilkins RB, Pressly JP. Diagnosis and incidence of lacrimal calculi. Ophthalmic Surg 1980; 11: 787-9.

3 Jones LT. Tear sac foreign bodies. Am J Ophthalmol 1965; 60: 111-3.

4 Spaeth GL. Nasolacrimal duct obstruction caused by topical epinephrine. Arch Ophthalmol 1967; 77: 355-7.

Accepted for publication 24 September 1987. 